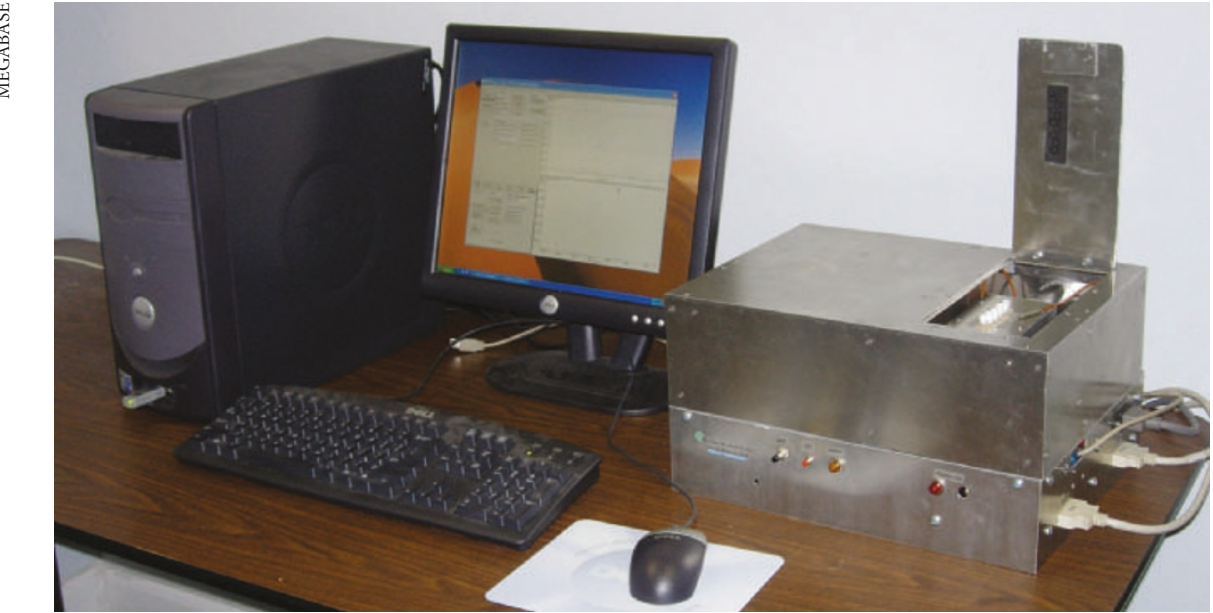

The PCRJet from MegaBase gives fast PCR with large samples.

more than 384 wells, more manufacturers are now making 1,536-well plates, including Corning, of Corning, New York, evotec technologies of Hamburg, Germany, and KBiosciences of Hoddesdon, UK. The problem with the larger plates is getting robotic support, but Victor Crew, sales manager at KBiosciences, says that it is possible to modify automated pipettors like the PlateMate from Matrix Technologies in Beverly, Massachusetts, to use them. The Equator HTS and Latitude pipetting systems from Deerac Fluidics in Dublin, Ireland, will also take 1,536-well plates and will fill one in less than 15 seconds.

Throughput can also be increased by multiplexing — running more than one spe- cific amplification reaction in a single tube. One approach is to use different colours of fluorigenic dyes to detect the different products. The new Mx3005P real-time PCR system from Stratagene of La Jolla, California, allows five different fluorigenic dyes to be used simultaneously. Using the company's FullVelocity probe-based real-time reagents, the machine will complete a 40-cycle twostep real-time PCR reaction in about 50 minutes. Cepheid of Sunnyville, California, make a real-time thermal cycler with fourcolour optics and 96 independently programmable reaction holders that claims to get the job done in as little as 20 minutes.

There is probably a limit to how many dyes can be added to a single reaction tube.
"Once you have got to four colours you have four sets of primers and four probes, and you have to stop these cross-reacting with each other and forming primer dimers," says Chris Helps from the School of Clinical Veterinary Science at the University of Bristol. "When you increase the number of targets the reactions become very complex - you also need spectrally distinct dyes to minimize cross-talk between channels."

\section{Blowing hot and cold}

How about really cutting the time down? The RapidCycler thermal cycler from Idaho Technology of Salt Lake City, Utah, blows blasts of hot and cold air through the reaction chamber, which gives near instantaneous temperature changes and rapid heat exchange with the samples. The RapidCycler 2 will do a 30-cycle run in 15 minutes, carrying 48 samples in either glass microcapillary tubes or the standard $1.5-\mathrm{mm}$ reaction cuvettes that will also fit the widely used LightCycler, available from Roche Applied Science in Indianapolis, Indiana.

And it is possible to go faster still. The PCRJet thermocycler developed by a multidisciplinary team under the brand name MegaBase Research Products, in Lincoln, Nebraska, drives a mixture of hot and cold gas through the reaction chamber at 45 miles per hour. "The velocity of the air stream is so high that we are definitely in the turbulent region, which ensures that the heat transfer to the sample-containing capillary tubes is maximal. We can do 30 cycles of PCR with

\title{
AMPLIFYING THE SIGNAL
}

When target amplification is not needed, northern blotting and other methods based on nucleic acid hybridization are convenient for detecting and measuring specific RNAs. Such methods include the Quantikine range for measuring specific cytokine mRNAs from R\&D Systems of Minneapolis, Minnesota. The problem with direct hybridization is generating a strong signal from a few target molecules.

Chad Mirkin, director of the Northwestern University Institute for Nanotechnology, at Evanston, Illinois, thinks he has cracked the problem.

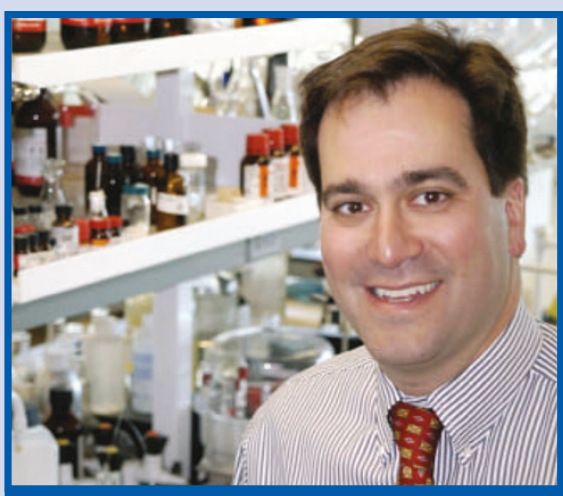

Chad Mirkin is barcoding proteins. $\mathrm{He}$ is co-founder of Nanosphere in Northbrook, Illinois, which produces BioBarcode, a sensitive detection system that can detect DNA sequences, but is proving to be just as good at spotting specific proteins. "PCR is amazing because it takes what you are trying to detect and duplicates a portion of it so that you have enough to detect. If you could do that for proteins, I would argue that you could have as big or a bigger impact, especially if you could do it without needing to use enzymes," he says.

Mirkin hopes to make that impact with BioBarcode, which works as a two-component immunological sandwich assay with some novel twists. Antibody molecules specific for one site on the target protein are tagged with 13-nm diameter gold nanoparticles, while larger paramagnetic particles are coated in a second antibody that recognizes a separate site on the target. If present, the target protein will grab both probes, and the complexes can be isolated by magnetic separation.

So far, not too unconventional. In Mirkin's system, however, the gold nanoparticle is smothered in hundreds or thousands of identical oligonucleotides, each strand hybridized to a short sequence that acts as a molecular barcode. Nanoparticles with different antibodies carry different barcodes, allowing multiplexing. After separation, the DNA barcodes are released and their identity determined by scanning on a biochip. The initial signal is thus amplified hundreds to thousands of times, allowing as few as 10 molecules of protein to be detected.

Randy Lewis at the University of Wyoming is using BioBarcodes to search for prions associated with the elk version of mad cow disease. "BioBarcodes give us an assay that is 1,000 times more sensitive than anything else," he says. "We should now be able to detect early onset and environmental contamination at lower levels than could be done before." 